\title{
Aging Skin: Nourishing from the Inside Out, Effects of Good Versus Poor Nitrogen Intake on Skin Health and Healing
}

\author{
Giovanni Corsetti, Evasio Pasini, Vincenzo Flati, Claudia \\ Romano, Anna Rufo, and Francesco S. Dioguardi
}

\section{Contents}

Introduction

Simple Steps and Methodology for Detecting and Monitoring Protein Malnutrition:

The First Step to Therapeutic Success

Static Biomarkers

A Functional Biomarker

Dynamic Biomarkers

Skin Aging, Collagen, and the Effects

of Malnutrition
Molecular Biologist's View of Skin Aging:

Intrinsic and Extrinsic Factors at Play

Evidence from Experimental Models of Essential Amino Acids in the Diet as a Key Factor in Maintaining the Integrity of Aged Skin and Accelerating Wound Closure

Conclusion ................................... 8

References $\ldots \ldots \ldots \ldots \ldots \ldots \ldots \ldots \ldots \ldots \ldots \ldots \ldots, 10$

G. Corsetti • C. Romano

Department of Clinical \& Experimental Sciences, Division of Human Anatomy and Physiopathology, University of Brescia, Brescia, Italy

e-mail: giovanni.corsetti@unibs.it

E. Pasini

"S. Maugeri Foundation", IRCCS, Cardiology

Rehabilitative Division, Medical Centre of Lumezzane,

Brescia, Italy

V. Flati • A. Rufo

Department of Biotechnological and Applied Clinical

Sciences, University of L'Aquila, L'Aquila, Italy

e-mail: vincenzo.flati@univaq.it

F.S. Dioguardi $(\varangle)$

Department of Internal Medicine and Community Health, University of Milan, Milan, Italy

e-mail: fsdioguardi@gmail.com 


\section{Abstract}

Skin is the outermost defense organ which protects us from the environment, constituting around $8 \%$ of an adult's body weight. Healthy skin contains one-eighth of the body's total proteins. The balance of turnover and synthesis of skin proteins is primarily dependent on the availability of sufficient nitrogen-containing substrates, namely, amino acids, essential for protein metabolism in any other tissue and body organs. The turnover of skin proteins has been shown to be rapid, and the mobilization of amino acids at the expense of skin proteins is relevant in experimental models of protein malnutrition. As a result, alterations in nutritional status should be suspected, diagnosed, and eventually treated for any skin lesions. Protein malnutrition has a dramatic prevalence in patients aged $>70$ or more, independent of the reason for hospitalization. The quality of nutrition and content of essential amino acids are strictly connected to skin health and integrity of its protein components. Collagen fiber deposition is highly and rapidly influenced by alterations in the essential to nonessential amino acid ratios. The most relevant nutritional factor of skin health is the prevalence of essential amino acids.

\section{Introduction}

Skin is an organ consisting of around $8 \%$ of an adult's body weight and is the outermost defense organ that protects us from environment. The skin is composed of the dermis and epidermis. The dermis is the inner layer and it has a mesodermic origin. The epidermis (epi = over) is the outermost layer of the skin, and it has an ectodermic origin [1]. The dermis provides all nutrients and oxygen to the epidermis through blood and lymph vessels. Thus, the oxygen and nutrition of the epidermis are provided from the internal to the external environment of the body (from the inside $o u t$ ), which should be borne in mind when considering skin health and its relationship with nutrition.
Collagen is ubiquitous in all organs and the most abundant protein in the human body $(30 \%$ of all body proteins) and represents around $50 \%$ of skin weight. Functionally, collagen maintains skin integrity by continuous remodeling stressed structures, so consuming a high amount of both energy and substrates for protein metabolism [2]. Indeed, collagen synthesis requires around four high-energy bonds ( ATP equivalents) for each amino acid inserted into the molecule [3]. Similar costs have been calculated for protein synthesis only in bacteria [4]. Therefore, the costs and complexity of wound healing (lesions of the epidermis and dermis from outside in) are an enormous metabolic problem, as discussed in chapter " $\gg$ Aging skin. Nourishing from Out-In. A Lesson from Wound Healing" in this textbook.

Consequently, the relationship between body protein and skin protein metabolism should be considered closely interlinked. The impairment of body protein metabolism would reflect similar impairment of skin health particularly in aged patients as will be discussed later. This is why treatment of wounds, especially chronic ones, solely by topical treatment, is only marginally effective. Therefore, before any procedure, dermatologists should assure that the patient's nitrogen/protein metabolism is supported adequately; otherwise, its local care, although potentially beneficial, could be ineffective.

Although alterations of skin structure are visible features of aging, there is only incomplete knowledge about the relation between normal or altered diet and skin aging, in particular when compared with the huge amount of published literature dealing with aging, diet, and peripheral muscle maintenance [5-7].

Healthy skin contains one-eighth of the body's total proteins. In addition, it contains collagen, constituting $70 \%$ of the total nitrogen content of the skin. So, the hypothesis that alterations of protein synthesis linked to malnutrition may drive alterations of the skin's extracellular matrix structure [8], as observed in many other organs, also makes sense in humans. As such, the collagen anabolic/catabolic ratios and balances may be strictly linked, and both skin and bone collagen 
health is coordinated by the whole-body protein synthesis balance, particularly in the elderly.

\section{Simple Steps and Methodology for Detecting and Monitoring Protein Malnutrition: The First Step to Therapeutic Success}

Early identification and evaluation of protein metabolic impairment are fundamental steps for better patient care, avoiding additional and independent damage and allowing traditional therapy to work properly. Assessment by specific biomarkers of early changes in body protein metabolism may be fundamental in identifying and monitoring initial damage and protein metabolic-related abnormalities before they are irreversible [9].

The first step in assessing global metabolic impairment is to evaluate body mass index (BMI). However, BMI cannot distinguish lean muscular from fatty tissues separately, and it is also influenced by fluid retention. Thus, body global protein metabolism can be more reliably measured by static, functional, and dynamic biomarkers. Indeed, we believe that a single ideal biomarker of protein metabolism impairment does not exist at this time. Each measurement has certain advantages, disadvantages, and/or limitations. It is recommended to use an integrated critical evaluation for best therapeutic success.

\section{Static Biomarkers}

These include: (1) anthropometric measurements, which distinguish muscular lean mass from fatty mass, and (2) visceral blood proteins, which provide information on visceral protein synthesis:

1. Anthropometric Measurements. The gold standard equipment for anthropometric measurements of body composition is the dual-energy X-ray absorptiometry (DEXA or DXA). However, DXA scanners are expensive equipment, and although DXA uses low-energy X-ray, patients are still exposed to radiation.
Skinfold thickness and arm muscle area are simpler methods for analyzing body composition and indirectly evaluating general protein metabolism. These biomarkers can be routinely evaluated at the patient's bedside, so specific training is not needed $[10,11]$ :

- Skinfold Thickness. This is an index of fatty mass. It should be measured using a plicometer in specific body sites such as the triceps (TSF), measuring along the midline on the back of the triceps of the right arm pinching the skin so that the fold is running vertically; pectoral, using a line from the fold of the axillary to the nipple, determining the midpoint; abdominal, measuring about $1 \mathrm{in}$. laterally to the right side, from 0.5 in. below the umbilicus lifting a horizontal fold of skin; suprailiac, measuring the top of the iliac crest; and thigh, using a midline of the front of the thigh and measuring midway between the inguinal crest.

- Arm Muscle Area (AMA). This is an index of lean muscular mass. First, we have to measure the mid-arm muscle circumference (MAMC). Then, the AMA can be calculated also using TSF according to the following formula:

$$
\begin{aligned}
\operatorname{MAMC}(\mathrm{cm})= & \operatorname{MAC}(\mathrm{cm})-3.14 \\
& \times \operatorname{TSF}(\mathrm{mm}) / 10 \\
& -[3.14 \times \operatorname{TSF}(\mathrm{mm}) / 10]
\end{aligned}
$$

TSF and AMA can be used in patients with fluid retention [12].

2. Blood Visceral Protein Assessment. Albumin, transferrin, prealbumin (or TTR), and retinolbinding proteins (RBP) are the most commonly used serum proteins. They have different blood half-lives with different turnovers [13].

- Albumin. Serum level of albumin $<3.5 \mathrm{~g} / \mathrm{l}$ is considered a biomarker of highly reduced protein metabolism. Concentrations less than $3.2 \mathrm{~g} / \mathrm{l}$ should be considered a biomarker of severe impairment, possibly 
associated with the onset of cachexia. Albumin has a blood half-life of about 20 days; consequently, it is not a rapid biomarker of successful protein synthesis after nutritional intervention, and it can be influenced by several diseases, such as severe nephrosis, protein-losing enteropathy, liver insufficiency, and fluid retention.

- Transferrin. This correlates with a mortality risk in hospitalized patients. It has a blood half-life of about 8 days, and this makes transferrin useful also for monitoring the medium-term effects of specific nutritional therapies.

- Prealbumin (Transthyretin, TTR). This has a plasma half-life of only about 24-36 h, so it responds quickly to changes in protein metabolism.

- Retinol-Binding Protein. This has a turnover of about $12 \mathrm{~h}$. Therefore, it is used to monitor rapid changes of protein metabolism. It is commonly available only in specialized laboratories.

\section{A Functional Biomarker}

The lymphocyte blood count is an indirect functional biomarker of cell proliferation, protein synthesis, and energy availability. Reduced lymphocyte protein metabolism causes cell-cycle loss with consequent low counts of circulating lymphocytes. Scarce circulating lymphocytes induce the malfunction of systemic immunity with an increased risk of infection [14]. This biomarker is easy to monitor but it should be interpreted together with other information.

\section{Dynamic Biomarkers}

1. Nitrogen Balance (NB). NB is the ratio of nitrogen introduced into an organism from consumed food AAs to the quantity of nitrogen excreted in the urine. NB is expressed as $\mathrm{g} /$ day according to the formula:
$\mathrm{NB}=$ Nitrogen intake $(\mathrm{NI})$

- Nitrogen output by urine $(\mathrm{NV})+2 \mathrm{~g}$

where:

$\mathrm{NI}=$ nitrogen intake/supply in $\mathrm{g} / \mathrm{day}^{-1}$ evaluated by protein intake ( $\mathrm{g} /$ day) where $\mathrm{g}$ of $\mathrm{N}$ is equal to intact protein $/ 6.25 \mathrm{~g}$.

$\mathrm{NV}=$ urinary nitrogen excretion as urea in g/day ${ }^{-1}+20 \% \mathrm{NV}$ for non-urea $\mathrm{N}$ excretion.

$2 \mathrm{~g}$ is the nitrogen lost in feces and sweat.

If NB $>1 \mathrm{~g} /$ day $^{-1}$ indicates the prevalence of protein synthesis, then $\mathrm{NB}<1 \mathrm{~g} /$ day $^{-1}$ suggests the prevalence of protein degradation [15].

NB is neither easy to obtain or to calculate. Moreover, NB depends mostly on urea concentrations as has recently been indicated. Urea concentrations in plasma and urine critically depend on the arginine content of diets, as arginine is the precursor of urea synthesis, cleaved by arginases forming urea and ornithine mostly but not exclusively by the liver. Arginase-1 (in the liver and erythrocytes) is rapidly inducible by high arginine content in diets. These modifications have multiple consequences; the misleading achievement of sufficient essential amino acid intake by a positive NB due to rapid elevation of plasma and urinary urea is just a part of this very complex clinical picture [16].

2. 3-Methylhistidine (3-MeH). 3-MeH is an index of proteolysis. 3-MeH originates from methylation of histidine. This process is stimulated by catabolic hormones, inflammatory molecules, and/or insufficient qualitative and quantitative nutrient intake including AAs [17]. Therefore, the massive presence of $3-\mathrm{MeH}$ in the blood or urine would suggest protein degradation. 3-MeH measurement is not easy to perform, and its value should be integrated with other biomarkers and indicators.

\section{Skin Aging, Collagen, and the Effects of Malnutrition}

The worst is not/ so long as we can say 'This is the worst'. (W. Shakespeare, King Lear, 4.1.29-30 Edgar) 
Collagen makes up for $70 \%$ of the total nitrogen content of the skin, and its fractional synthesis rate is relatively high in both animals and humans. Indeed, the collagen synthesis rate is superior to $56-72 \%$ a day in the epidermis and $2.6-2.9 \%$ in the dermis [18]. Furthermore, rapid transportation of nutrients between the dermis and epidermis has been found in healthy well-fed patients, able to maintain epidermal integrity.

With the progression of age, the skin undergoes a series of events that gradually reduce its structural integrity and function with changes of appearance and structure. As already observed, the dermis owes its structural stability and resilience to collagen, so the reduction and/or alteration of collagen proteins leads to changes of the appearance and function of the skin. The quality of the collagen that remains is altered by greater disorder. Like collagen, elastin displays morphology alterations, which decreases the elasticity of the skin in the aged dermis [19]. It is interesting to note that skin collagen and bones as well as connective tissue of most body organs have similar amino acid sequences typical of collagen type I.

The functional, age-related, changes of the skin include slowing of reepithelialization. Thus, the skin becomes more fragile and thin, and in the elderly, skin wounds need more time to complete healing. However, the clinical impact of these changes in acute wound healing of healthy people seems to be small. On the contrary, poor healing in chronic wounds is largely seen in the elderly with comorbidities [20].

Histological analysis of skin thickness shows that the dermis of malnourished animals is significantly thinner compared to well-nourished animals [8]. This observation is concomitant with compromised collagen synthesis in other organs such as the intestinal wall [21]. These findings indicate that skin collagen integrity is strictly entangled with protein malnutrition. Clinically, this has been proven by the impaired growth in malnourished children [22] and the importance of implementing an adequate protein intake in the elderly to maintain bone health [23].

It is also interesting to note that malnutrition impairs skin vitamin $\mathrm{D}_{3}$ production. Interestingly, vitamin $\mathrm{D}_{3}$ is involved in anabolic processes reducing other aspects of body metabolic efficiency $[24,25]$. Therefore, the skin of aging people may pay debts that are shared with all other body organs, so the skilled dermatologist should be able both to detect protein malnutrition and to solicit adequate nutrition protocol. If not, the topical therapeutic approach will not be efficient. Finally, there are many different molecular mechanisms connected with skin aging [26]; the most representative are described below.

\section{Molecular Biologist's View of Skin Aging: Intrinsic and Extrinsic Factors at Play}

It is well known that skin aging is a consequence of genetically determined biological factors influenced by oxidative stress $[27,28]$ and environmental (such as sunlight ultraviolet radiation) factors [29]. The first type of skin aging is called intrinsic aging, while the second is called extrinsic aging [30, 31]. Nevertheless, it is reasonable to think that extrinsic aging works on the same basis as the intrinsic mechanisms of aging.

The intrinsic aging process is thought to be similar to that occurring in internal organs. The external stratum corneum seems to be unaffected, while the epidermis and dermis lose their function and structure. Indeed, a reduced number of fibroblasts and a reduction of their biosynthetic capacity have been observed. Furthermore, there is an increase in the density of collagen fibers that are also randomly oriented [26].

The main molecular determinants of the skin aging process include reduced skin cell proliferation capacity, reduced collagen type I synthesis, and increased collagen breakdown mediated by matrix metalloproteinase (MMP) activity, further promoted by a concomitant decrease of MMP inhibitors. The result is the alteration, structural and compositional, of the long-lived collagen types I and II, which are the main structural components of the extracellular matrix proteins of the dermis [32]. Indeed, it has been shown that changes of the epidermis are minimally involved with skin aging, while in major 
alterations involving the dermis, there can be seen damaged and disorganized collagen fibrils.

Furthermore, a reduced proliferative rate of skin cells (fibroblasts, keratinocytes, melanocytes) is also found with aging. These changes tip the balance from matrix-producing to matrixdegrading phenotype, so determining atrophy of the dermis and so the occurrence of wrinkles. Nevertheless, the molecular mechanisms underlining the intrinsic and the extrinsic skin aging, although sharing similarities, are different with MMP activity, particularly on photoaged skin.

At the molecular level, it has been shown [33] that ultraviolet radiation (probably the most important factor responsible for extrinsic skin aging) is capable of modulating MMP induction through the activation of growth factor receptors on fibroblasts and keratinocytes. The activated epidermal growth factor receptor (EGFR), through the GTP-binding regulatory protein p21Ras, mediates the activation of the MAP kinase signal transduction, which activates ERK, JNK, and p38MAPK. Both JNK and p38MAPK phosphorylate and activate the activating transcription factor 2 (ATF2) that upregulate c-Jun expression. This associates with the constitutively expressed c-Fos to form the activator protein (AP) 1 transcription factor which is necessary for the transcription of MMP genes. c-Jun expression is elevated in the old skin. Indeed, in human skin, the AP-1 transcription factor activity is limited by the expression of c-Jun being c-Fos expressed at the same rate in the young and in the old skin.

Other growth factor receptors, such as interleukin-1 receptor, tumor necrosis factor receptor, and platelet-derived growth factor receptor, also share this action mechanism. The common endpoint is the loss of collagen in the skin with a remodeling of elastic fibers. This event is modulated differently in extrinsic and intrinsic aging and is associated with the loss of tissue compliance and resilience and the formation of wrinkles. It therefore seems that there is a causative link between elastic fiber remodeling and functional changes (loss of elasticity) in aging skin.
Studies conducted by several groups have revealed that UV radiation mainly increases three MMPs, that is, the collagenase MMP1, the stromelysin-1 MMP3, and the gelatinase MMP9. All of these are regulated at the transcriptional level by the AP-1 transcription factor. These MMPs are induced in the epidermis (epidermal keratinocytes) and are secreted in the dermis where they degrade collagen. It has been hypothesized that dermal cells might also be involved with MMP production through a paracrine mechanism mediated by the release of growth factors or cytokines, which in turn modulate MMP production in epidermal keratinocytes [34, 35].

Fragments of elastin and fibrillin-1 deriving from elastic fiber remodeling are capable of influencing the expression of MMPs and, as a consequence, promoting the degradation of most dermal proteins such as collagen. Furthermore, fibrillin may play a role in mediating homeostasis of the tissue by mediating the sequestration of TGF $\beta$ [36-38]. TGF $\beta$ is a tightly regulated growth factor that controls cell proliferation, survival, differentiation, and migration. Its latent and inactive form is non-covalently bound to other proteins such as the latency-associated pro-peptide (LAP) to form the small latent complex (SLC), and when bound by latent TGF $\beta$-binding protein (LTBP), it forms a large latent complex (LLC).

LTBPs are proteins structurally related to fibrillins that regulate TGF $\beta$ activity. It has been suggested that the activation of the latent TGF $\beta$ is mediated by its proteolytic release from the inactive latent complex, by the competition of thrombospondin-1 with SLC, by $\mathrm{pH}$ changes, or by the action of reactive oxygen species. The active TGF $\beta$ then binds to the TGF $\beta-R 1$ and TGF $\beta$-R2 heterodimer, which leads to the phosphorylation of the TGF $\beta$-R1, which in turn phosphorylates the intracellular signaling proteins SMAD2 and SMAD3. Their phosphorylation allows the binding of another intracellular protein called SMAD4, which in turn allows the translocation of the complex into the nucleus where it acts as a transcription factor (in association with cofactors) for the regulation of target-gene expression. Fibrillin-1 has been shown to mediate TGF $\beta$ 
release from the inactive latent complex, thus promoting its activity. The impairment of the TGF $\beta$ pathway is therefore probably responsible for the reduction of procollagen synthesis in aged skin.

Since dryness and the associated loss of elasticity are also associated with skin aging, a further important mediator is the skin's capacity to retain water in order to maintain skin moisture. The key molecular mediator involved with skin moisture maintenance is hyaluronic acid (HA). This is a glycosaminoglycan mainly expressed in the extracellular matrix of the skin. It is synthesized by HA synthases (HAS), while it is degraded by hyaluronidases. The rate of HA turnover is dynamic, and in the skin, it has a half-life less than a day. TGF $\beta$ is an important stimulator of HA synthesis since the expression of HAS1 and HAS2 is regulated by TGF $\beta$, although differentially in the dermis and epidermis. The marked reduction of HA in aging skin is associated with its loss in the epidermis, while it is conserved in the dermis. Furthermore, a reduction in size of the HA polymers in the skin [39] has also been observed.

Extrinsic skin aging is also characterized by a distinct homeostasis of HA [40]. Photo-exposed skin shows an increased amount of degraded (lower molecular weight) HA compared with the photo-protected skin, and this degradation is associated with an increase of hyaluronidases 1,2 , and 3 and with a decreased expression of HAS1. This underlines differences at a molecular level which distinguish photoaging (extrinsic) from natural aging (intrinsic).

\section{Evidence from Experimental Models of Essential Amino Acids in the Diet as a Key Factor in Maintaining the Integrity of Aged Skin and Accelerating Wound Closure}

Amino acids (AAs) are known to regulate protein metabolism, and they have been tested in order to establish their effect on the dermal tropocollagen synthesis rate. It has been observed that a combination of branched-chain AA (BCAA) and glutamine or proline derived from glutamine metabolism is important for restoring the impaired dermal collagen protein synthesis in malnourished animals [41].

It is accepted that protein malnutrition is a well-established factor damaging skin health. However, the role and relevance of peculiar amino acids in skin health and improvement of wound-healing processes are not clear. Indeed, previous results showed that the excess of nonessential AA (NEAA) provokes negative effects on protein synthesis and cannot be considered "nonbelligerent" in protein metabolism. Indeed, an excess of NEAA impairs wound health and healing (see also chapter " $>$ Aging Skin. Nourishing from Out-In. A Lesson from Wound Healing"). Thus, in order to study the role and effects of the availability of different percentages of EAA and NEAA, an experimental pilot study in healthy middle-aged rats (12 months) compared the effects of three different feeds on wound healing, each of which contained a different ratio of EAA to NEAA, while nitrogen content remained strictly the same. The three groups (identified in images as A, E, and D) had the following percentage of $\mathrm{AA}$ as components of nitrogen intake: (1) diet A contained exclusively EAA (100\%); (2) diet E contained $45 \%$ of EAA and $55 \%$ of NEAA, which is the same ratio found in casein (used as control group); and (3) diet D contained predominantly NEAA (85\%) while EAAs were only $15 \%$.

Preliminary data showed that already after 15 days, there was a marked difference in body weight (b.w.) between the three groups. In particular, the tendency to reduce b.w. in group D compared to an increase in group E. After 15 and 60 days of treatment, b.w. difference between animals fed with different diets varied significantly. In particular, diet D caused a steady and rapid decrease of weight, reaching $28 \%$ after 60 days. By contrast, with diet E, there was an increase of about $12.5 \%$ in b.w., while diet A did not lead to any significant changes in b.w., although growth in length was not affected and there was evident loss of abdominal fat, with only $6 \%$ of increases in b.w. after 60 days. Curiously, the animals fed with the diet $\mathrm{D}$, although they had a considerably reduced b.w., ate about $23 \%$ more 
than the control group and drank about $50 \%$ more than diet E-fed animals.

The physical impairment of the animals was also accompanied by early alteration of skin structure. Feeding with diet D reduced the thickness of the epidermis and altered cellular organization. The basal layer was formed by abnormal cells with flattened and elongated nuclei, while the granular layer was absent or very reduced (Fig. 1a-c). Moreover, a diet deficient in EAA also changes the organization of the dermis. In fact, with diet $\mathrm{D}$ the collagen fibers became thinner and more spaced and tended to arrange themselves in a more disorganized manner (Fig. 1d-f).

The effect of the different relations between EAA and NEAA was also observed in the time needed to heal identical wounds. The wounds produced in animals fed with diet A, composed mainly of EAA, healed quicker compared to the standard diet E, based on casein and therefore containing about $55 \%$ of NEA. In contrast, the wounds of the animals fed with diet $\mathrm{D}$, poorest in EAA ( $85 \%$ of NEA), had significantly greater healing times (Fig. 2).

The different timespans of wound closure also seem to be due to the fact that the availability of EAA affects the production and organization of collagen fibers. Indeed, already after 15 days from the wound, with diet A, abundant production of thick and well-oriented collagen fibers was observed. On the contrary, diet D produced scarce and thin collagen fibers with random orientation (Fig. 3a-c).

Similarly, after 30 days from wounding, although wounds were healed for all groups, the organization and thickness of the collagen fibers were largely different (Fig. 3d-f). These observations suggest that it is not the amount of nitrogen, but the quality of the nitrogen sources that affects the physical condition. Although the study is still preliminary, physical exhaustion and morphological and structural changes of the skin seem comparable with those observed during senescence. It is therefore possible that the prevalence of EAA in the diet may be a key to prevent or slow tissue aging, confirming the observations by D'Antona et al. [42] in muscles and the heart. However, preliminary data also provide another aspect until recently little known, i.e., that NEAA significantly hinders the normal cell physiology in a dose-dependent manner. This was particularly evident in influencing the timing of wound healing. In accordance with this, as reported in another chapter " $>$ Aging Skin. Nourishing from Out-In. A Lesson from Wound Healing," NEAA significantly hinders the normal cell physiology in a dose-dependent manner since they are particularly efficient in influencing the timespan of wound healing.

We believe that these findings may open new scenarios in understanding the causes that lead to the impairment of health of aging skin, as well as suggesting procedures for the prevention or treatment of related pathologies.

\section{Conclusion}

Collagen metabolism is fundamental for maintaining skin integrity as it makes up $70 \%$ of total nitrogen content of skin proteins. However, skin collagen metabolism is negatively altered in protein-malnourished states often present in aged people. The alterations of skin linked to protein metabolism impairment are an aspect of human pathophysiology, although not easily evaluable, this is worth attention and the development of the specific procedures of assessment of damages and success of therapies. Indeed, insufficient amino acids or altered balance between EAA and NEAA modifies the balance of collagen protein turnover and resynthesis. Although there is debate about which is the best formulation of amino acids suitable for maintaining and promoting skin collagen health, certainly a primary role is linked to sufficient essential amino acid availability. This is not an isolated requirement of skin, but an obliged requirement of skin in a complex balance ruled by metabolism requirements of the whole body's tissues and organs. Consequently, 

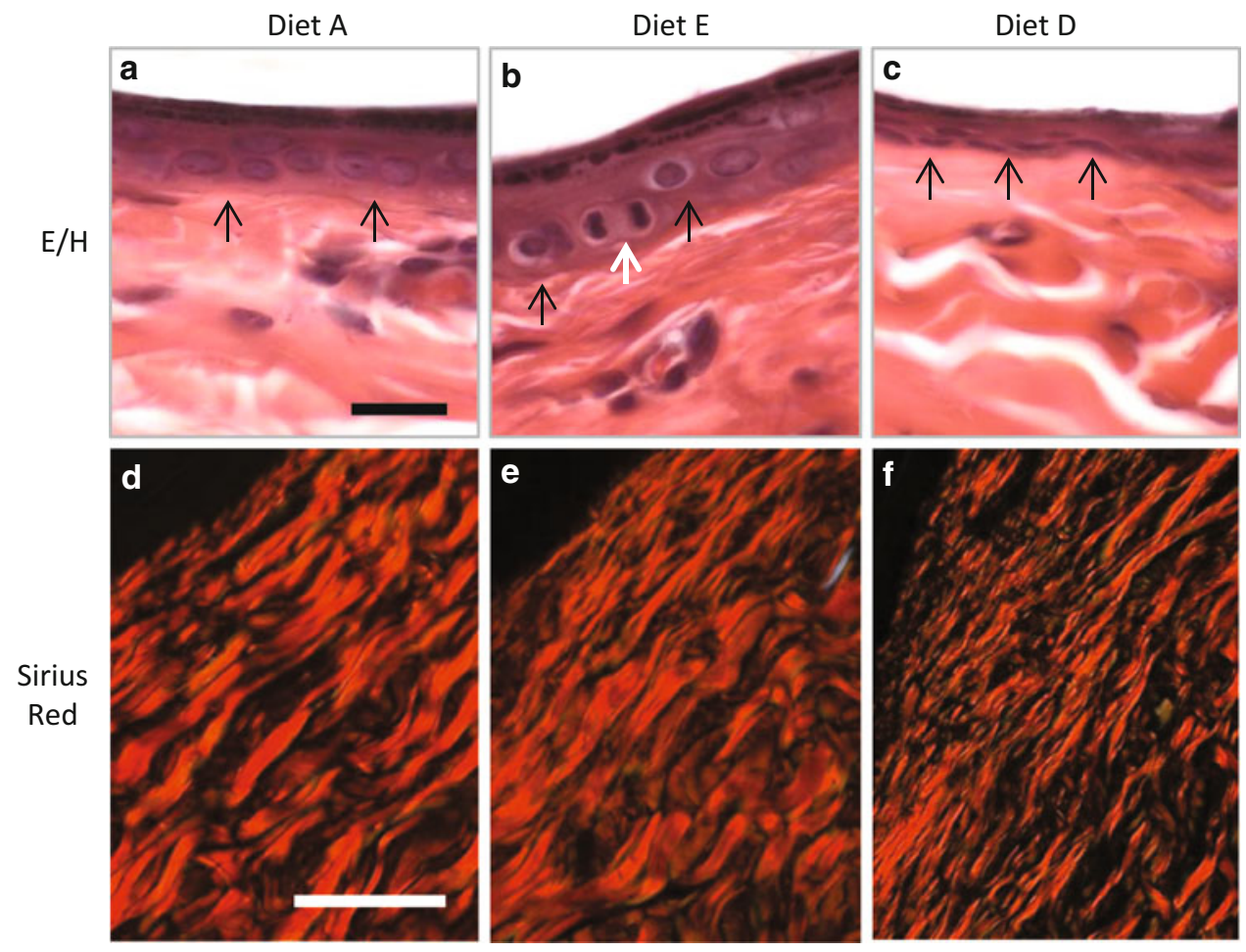

Fig. 1 (a, b). Eosin-hematoxylin staining. Epidermis after 60 days of treatment with the diets. (a) With diet A, the organization of the epidermis and cell morphology of basal layer (arrows) are regular. (b) With diet E, the organization of the epidermis does not undergo significant changes; the basal layer frequently shows duplicating cells (white arrow) and more mature cells without cytoplasm (black arrows). (c) Diet D reduces the thickness of the epidermis and alters the cellular organization. The basal layer is formed by abnormal cells with flattened and elongated nuclei (arrows), while the granular layer is absent or very reduced. Scale bar $150 \mathrm{um}$. (d, e). Sirius red stain for collagen (polarized light). The organization of collagen in the dermis changes with the type of diet. (d) With diet A, the collagen fibers are thicker and more compact. (e) With diet $\mathrm{E}$, the collagen fibers do not seem to vary significantly; however, the tendency to thinning is observed. (f) With diet $\mathrm{D}$, the collagen fibers become thinner and more spaced and tend to arrange themselves in a more disorganized manner. Scale bar 300 um (Corsetti et al.)
Fig. 2 Wound area according to diet. All wound areas were converted to $100 \%$ on the day of wounding (day 0 ). On subsequent days $(3,6$, 15 , and 30$)$, the areas were expressed as a percentage of original area on day zero. $*$ and ${ }^{\circ}=\mathrm{p}>0.05$ versus $\operatorname{diet} \mathrm{E} . \wedge=\mathrm{p}>0.05$ versus diet $\mathrm{D}$ (Corsetti et al.)

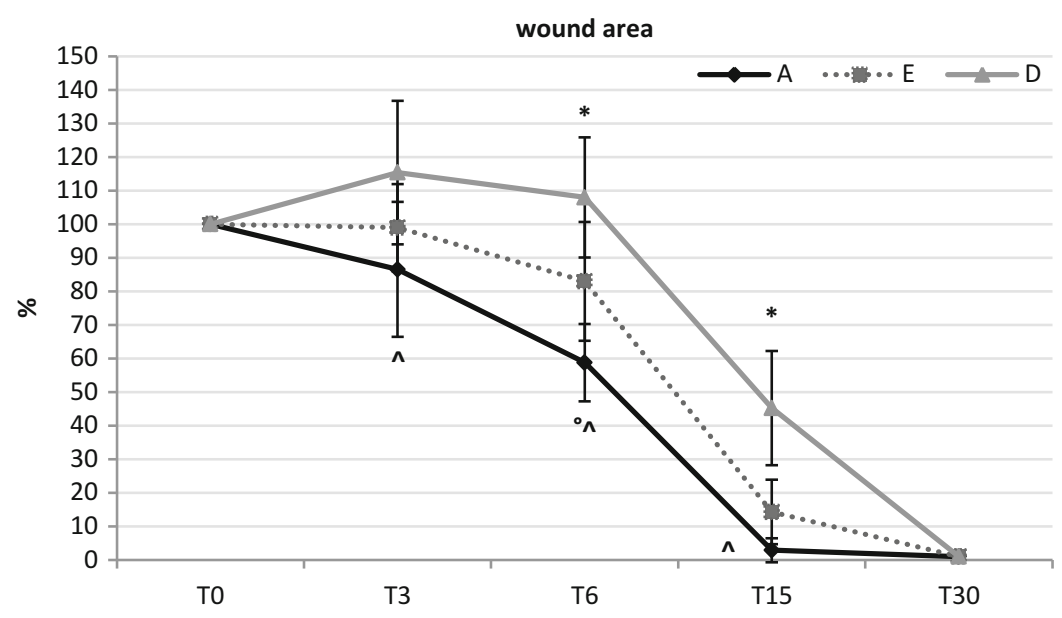



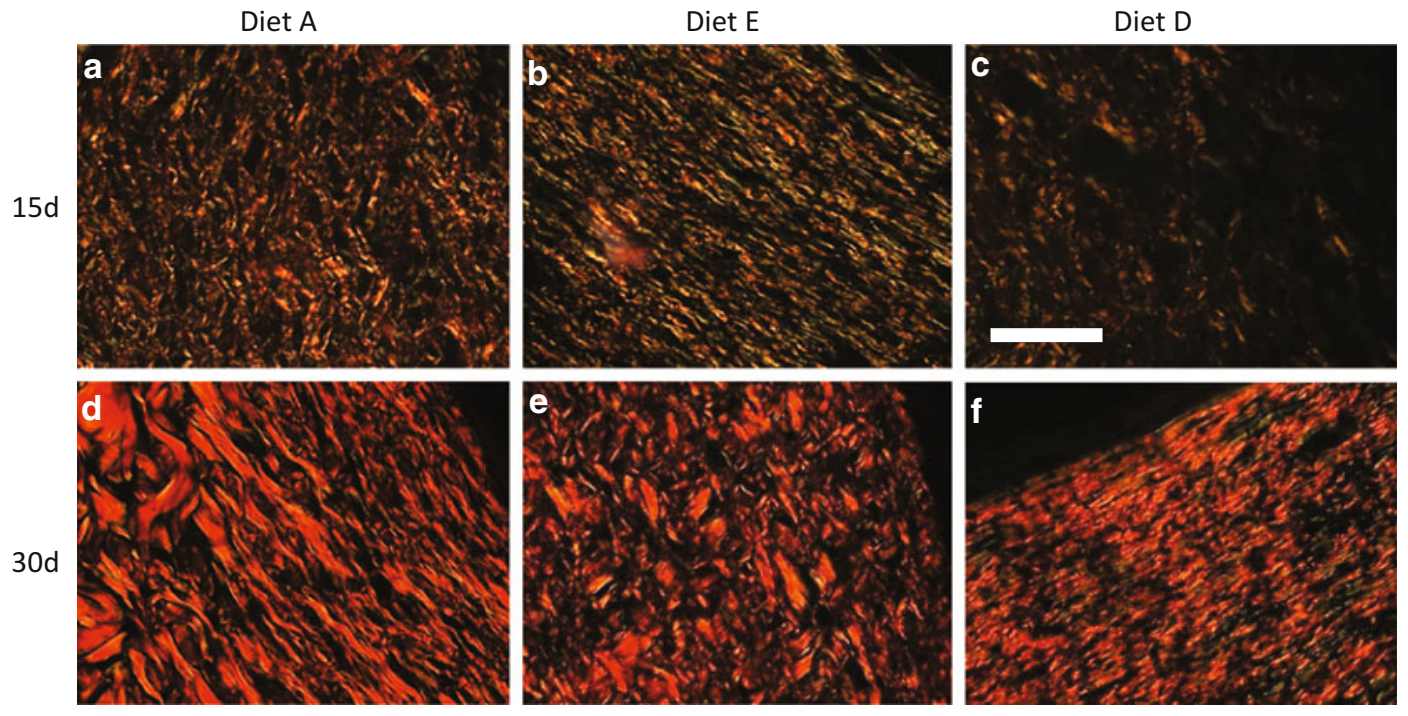

Fig. 3 Wound area. Sirius red stain - polarized light. Collagen fibers in the upper layers of the newly formed tissue in the wound area after $15(\mathbf{a}-\mathbf{c})$ and 30 days $(\mathbf{d}-\mathbf{f})$ according to diets. The different thickness and quantity of

matching the needs of sufficient essential AA intakes is important for the body's organ metabolism and the skin's needs should be included in these total requirements.

\section{References}

1. Xua Z, et al. Teleost skin, an ancient mucosal surface that elicits gut-like immune responses. Proc Natl Acad Sci U S A. 2013;110(32):13097-102.

2. Xiao-Jun Z, et al. Measurement of protein metabolism in epidermis and dermis. Am J Physiol Endocrinol Metab. 2003;284:1191-201. doi:10.1152/ ajpendo.00460.2002.

3. Browne GJ, Proud CG. Regulation of peptide-chain elongation in mammalian cells. Eur J Biochem. 2002;269:5360-8. doi:10.1046/j.1432-1033.2002. 03290.x.

4. Kaleta C, et al. Metabolic costs of amino acid and protein production in Escherichia coli. Biotechnol J. 2013;8:1105-14. doi:10.1002/biot.201200267.

5. Kim J, et al. Association between healthy diet and exercise and greater muscle mass in older adults. J Am Geriatr Soc. 2015;63(5):886-92. doi:10.1111/ jgs. 13386.

6. Paddon-Jones D, et al. Protein and healthy aging. Am J Clin Nutr. 2015;101:1339S-45. doi:10.3945/ ajcn.114.084061. the collagen fibers in the diet $\mathrm{D}$ were observed compared to the other diets, as well as the disorganization of fibers after 15 and 30 days from the wound. Scale bar 100 um (Corsetti et al.)

7. Murphy $\mathrm{CH}$, et al. Hypoenergetic diet-induced reductions in myofibrillar protein synthesis are restored with resistance training and balanced daily protein ingestion in older men. Am J Physiol Endocrinol Metab. 2015;308:E734-43. doi:10.1152/ajpendo.00550.2014.

8. Leite SN, et al. Experimental models of malnutrition and its effect on skin trophism. An Bras Dermatol. 2011;86(4):681-8.

9. Pasini E, et al. The enemy within. How to identify chronic diseases induced-protein metabolism impairment and its possible pharmacological treatment. Pharmacol Res. 2013;76:28-33.

10. Cogill B. Anthropometric indicators measurement guide. Washington, DC: Food and Nutrition Technical Assistance Project, Academy for Educational Development; 2001.

11. Magnani R. Sampling guide. Arlington: Food Security and Nutrition Monitoring (IMPACT) Project, ISTI, Inc., for the U.S. Agency for International Development; 1999.

12. Frisancho AR. Anthropometric standard for assessment of growth and nutritional status. Ann Arbor: University of Michigan Press; 2004. p. 38-62. ISBN 0-472-10146-3.

13. Watson RR. Nutritional stresses: levels of complement proteins and their functions. In: Watson RR, editor. Nutrition, disease resistance and immune function. New York: Marcel Dekker; 1984. p. 175-88.

14. Acanfora D, et al. Relative lymphocyte count: a prognostic indicator of mortality in elderly patients with congestive heart failure. Am Heart J. 2001;142 (1):167-73. 
15. Aquilani R, et al. Is nutritional intake adequate in chronic heart failure patients? J Am Coll Cardiol. 2003;42:1218-23.

16. Dioguardi FS. Nutrition and skin. Collagen integrity: a dominant role for amino acids. Clin Dermatol. 2008;26 (6):636-40.

17. Ferrari $F$, et al. A rapid method for simultaneous determination of creatinine, 1- and 3 methylhistidine in human urine. Electrophoresis. 2009;30:1-3.

18. Zhang XJ, et al. Measurement of protein metabolism in epidermis and dermis. Am J Physiol Endocrinol Metab. 2003;284:1191-201.

19. Lavker RM, et al. Aged skin: a study by light, transmission electron, and scanning electron microscopy. J Invest Dermatol. 1987;88:44S-51.

20. Thomas DR, Burkemper NM. Aging skin and wound healing. Clin Geriatr Med. 2013;29(2):xi-xx. doi:10.1016/j.cger.2013.02.001

21. Nakajima V, et al. Alterations in the intestinal wall due to protein malnutrition in rats: evaluation of the rupture strength and the tissue's collagen. Acta Cir Bras. 2008;23(5):435-40.

22. Allison SP. Malnutrition, disease, and outcome. Nutrition. 2000;16(7/8):590-3.

23. Beasley JM, et al. Biomarker-calibrated protein intake and bone health in the Women's Health Initiative clinical trials and observational study. Am J Clin Nutr. 2014;99:934-40.

24. MacLaughlin J, Holick MF. Aging decreases the capacity of human skin to produce Vitamin $\mathrm{D}_{3}$. J Clin Invest. 1985;76:1536-8.

25. Bikram S, et al. Nutritional deficiencies after bariatric surgery. Nat Rev Endocrinol. 2012;8:544-56.

26. Jenkins G. Molecular mechanisms of skin ageing. Mech Ageing Dev. 2002;7:801-10.

27. Poljsak B, et al. Intrinsic skin aging: the role of oxidative stress. Acta Dermatovenerol Alp Pannonica Adriat. 2012;21:33-6.

28. Rinnerthaler $\mathrm{M}$, et al. Oxidative stress in aging human skin. Biomolecules. 2015;5:545-89.

29. Farage MA, et al. Characteristics of the aging skin. Adv Wound Care. 2013;2:5-10.

30. Farage MA, et al. Intrinsic and extrinsic factors in skin ageing: a review. Int J Cosmet Sci. 2008;30:87-95.
31. Vierkotter A, Krutmann J. Environmental influences on skin aging and ethnic-specific manifestations. Dermatoendocrinol. 2012;4:227-31.

32. Fisher GJ, et al. Mechanisms of photoaging and chronological skin aging. Arch Dermatol. 2002;138:462-70.

33. Fisher GJ, et al. Retinoic acid inhibits induction of c-June protein by ultraviolet irradiation that occurs subsequent to activation of mitogen activated protein kinase pathways in human skin in vivo. J Clin Invest. 1998;101:1432-40.

34. Quan T, et al. Matrix-degrading metalloproteinases in photoaging. J Investig Dermatol Symp Proc. 2009;14:20-4.

35. Chauhan P, Shakya M. Modeling signaling pathways leading to wrinkle formation: identification of the skin aging target. Indian J Dermatol Venereol Leprol. 2009;75:463-8.

36. Ashworth JL, et al. Fibrillin degradation by matrix metalloproteinases: implications for connective tissue remodelling. Biochem J. 1999;340:171-81.

37. Kaartinen V, Warburton D. Fibrillin controls TGF-beta activation. Nat Genet. 2003;33:331-2.

38. Chaudhry SS, et al. Fibrillin-1 regulates the bioavailability of TGFbeta1. J Cell Biol. 2007;176:355-67.

39. Papakonstantinou E, et al. Hyaluronic acid: a key molecule in skin aging. Dermatoendocrinol. 2012;4:253-8.

40. Tzellos TG, et al. Extrinsic ageing in the human skin is associated with alterations in the expression of hyaluronic acid and its metabolizing enzymes. Exp Dermatol. 2009;18:1028-35.

41. Murakami H, et al. Combination of BCAAs and glutamine enhances dermal collagen protein synthesis in protein-malnourished rats. Amino Acids. 2013;44:969-76.

42. D'Antona G, et al. Branched-chain amino acid supplementation promotes survival and supports cardiac and skeletal muscle mitochondrial biogenesis in middleaged mice. Cell Metab. 2010;12:362-72. 